\title{
EL DERECHO EN LA HISTORIA: LAS SIETE PARTIDAS Y SU LUGAR COMO EVENTO CULTURAL
}

\author{
Daniel A. Panateri \\ Consejo Nacional de Investigaciones Cientificas y Técnicas (Conicet), Argentina \\ danielpanateri@conicet.gov.ar
}

\section{Introducción}

En el siglo XIII se produjeron cambios sustanciales en la producción cultural medieval. El reinado de Alfonso X tuvo lugar en ese siglo (1252-1284). Asimismo, el Rey Sabio colaboró con lo que hoy debiera ser visto como el siglo del derecho medieval. Más allá de las discusiones y posturas modernas, el propósito de mi análisis es considerar la posibilidad de conocer al rey a través de sus obras. Esas obras son los elementos que conectan de modo incesante con aquel rey que yace, su efigie, incólume sobre las escalinatas de la Biblioteca Nacional de Madrid como protector de la cultura y, quizá también, como propia categoría cultural. Sin olvidar que en noviembre de 2021 se cumplirán ochocientos años de su nacimiento, propongo un análisis del impacto de su obra jurídica más allá de las alusiones a su figura personal. En el espacio designado dentro del monográfico será imposible desarrollar las problemáticas y virtudes en torno a todas ellas, en especial reconstruyendo los aportes de la crítica. En ese sentido, propongo utilizar una tesis crítica a un modelo ya conocido para reflexionar en torno a la pervivencia, vitalidad y carácter de su obra legal más importante: las Siete Partidas $(=S P)$.

El modelo al que refiero es el que denominaré, sin originalidad, «corriente codificatoria». Mi planteo implica conocer este modelo, establecer las críticas, explicarlas y luego avanzar hacia la posibilidad de comprender por qué $S P$ no puede ser reducida a la idea de corpus iuris del siglo XIII. En este camino, mencionaré algunas nociones sobre el estado de otros corpora de 
ese momento (tarea esencial para avanzar sobre mi crítica teórica ya mencionada $^{1}$ y cuál entiendo sería la mejor calificación para la práctica cultural que subyace a la escritura del derecho alfonsí. Esta no será una intervención de defensa nominalista, sino una propuesta de comprensión no retrospectiva (dentro de los límites evidentes de dicha propuesta) a un fenómeno cultural del derecho a través de la historia.

\section{Corriente codificatoria en el siglo XIII: definición y crítica}

Desde mediados de la década de 1980 y hasta mediados de la década de 1990 asistimos a una serie de textos que propusieron una visión sobre el derecho y la política anclada en el presente desde el que escribían. Una práctica que buscaba respuestas para su tiempo a través de conjeturas sobre el pasado y de preguntas hacia él. Algunas de esas contribuciones poseen un valor inestimable tanto a nivel historiográfico como teórico ${ }^{2}$. Considero, con la distancia a mi favor, retomar críticamente esos postulados para aportar una lectura que sume al entramado interpretativo sobre un fenómeno complejo como fue la compilación de derecho por parte de las monarquías europeas, con especial énfasis en $S P$.

El núcleo de funcionamiento de este argumento que critico se cifra en dos elementos. Primero, la compilación de leyes durante el siglo XIII fue un fenómeno global (europeo) que conllevó un proceso de codificación legislativa, incluso sin atender a las diferencias de formato entre estas «codificaciones». Por ejemplo, no es igual el Vidal Mayor ${ }^{3}$ de 1247 que las Ordonnaces compiladas en la Reformation des moeurs de 1254, ni las Statutes de Eduardo I de 1267-99 o las Siete Partidas de Alfonso X. No son semejantes porque poseen finalidades distintas, así como, fundamentalmente, métodos de creación jurídica (y normativa) diferentes. Incluso aceptando la definición de código para el período, de los cuatro ejemplos el que más se acerca a uno es $S P$, mientras que desdeñaría de cuajo esa definición para los restantes ${ }^{4}$. El segundo elemento es uno tautológico y que se resume en la frase de Wolf (1993-94: 40):

\footnotetext{
${ }^{1}$ Para quien esté interesado en una comparación entre los diversos corpora: Wolf (1989).

${ }^{2}$ Algunos ejemplos pueden ser Wolf (1983 y 1993-94) y Black (1992), entre otros.

${ }^{3}$ Resumo con Vidal Mayor la tradición manuscrita de cuatro obras distintas -pero genéticamente relacionadas- como son los Fori Aragonum, Fueros de Aragón, Maior Compilatio y Vidal Mayor. Véase Delgado Echeverría (2009) y las referencias que expone para completar el panorama.

${ }^{4} \mathrm{Con}$ respecto a las críticas a las que hago referencia pero no desarrollo por cuestiones de espacio, Panateri (2019a y 2020) y Ascheri (2003), quien sostiene ocho puntos de separación ontológica entre el código, eminentemente moderno, y el derecho común, eminentemente medieval. Caroni (2013) retoma una crítica desde la perspectiva de la «mentalidad codificatoria» propia del siglo XVIII y que, como tal, no se encuentra sino como ejercicio retrospectivo de una realidad ya pasada para nosotros, pero posterior
} 
«Podemos decir, para la legislación a partir del siglo XIII: [1]os Estados territoriales en formación generaron leyes, porque las leyes generaban Estados territoriales». El apriorismo confeso, que puede ser útil en una formulación teórica con fines especulativos, conlleva el artificio de una proyección retrospectiva que condiciona la producción cultural jurídica medieval a una expresión inacabada del Estado moderno y su forma de establecer legislación ${ }^{5}$. Así, el fracaso de estas «codificaciones» se habría debido a la fuerza de los estamentos que se levantaron contra las monarquías modernizadoras ${ }^{6}$.

En este procedimiento se encuentran imbricadas una serie mayor de recurrencias y condicionantes históricos que surgen de la propia práctica científica. De algún modo, la idea de que incluso los iura propria conformaron un fenómeno propiamente jurídico único y común en un determinado período conforma un bloque donde Europa posee una entidad anudada por la expresión legal de "hacer leyes" bajo un determinado régimen que finalmente es siempre romano (con aire de corpus documental perfectamente definido e identificable). En ese punto, sea ius commune o ius proprium no es posible salir de la trampa que extirpa del derecho su condición cultural y su historia. Asimismo, es innegable que gran parte del contenido de estas compilaciones es romano, pero sostengo que el modo de composición, i.e. la práctica cultural que sustenta el libro de derecho alfonsí, imprime determinaciones semánticas y condicionantes de lectura que lo transforman en otra cosa.

El punto clave es cómo entender esas expresiones jurídicas en tanto manifestaciones culturales propias de un tiempo y lugar en que cosas parecidas suceden, aun sin ser lo mismo. Como decía Caroni (2013: 27):

en la historia del derecho se habla de código para aludir a muchos «productos» claramente diferentes y heterogéneos, lo que puede suscitar alguna perplejidad justificada. Cito algún ejemplo para demostrar que no exagero: el Código de Hammurabi (de 1700 a. C.), el Codex Justinianus (de 529), el Speculum saxonicum (Sachsenspiegel, de 1230), el Code Napoléon (de

al período medieval. Asimismo, desde una mirada de los estudios sobre la modernidad: Clavero (1991: 61-128) y Bermejo Castrillo (2013).

${ }^{5}$ Vale aclarar también que esa imagen del Estado moderno luce más decimonónica que de fines del siglo xx. En parte, creo que revitalizar esa imagen del poder público fue una reacción al progresivo, pero contundente, retroceso de esa misma imago frente al avance del mercado como lazo de las relaciones sociales a nivel global.

${ }^{6}$ Sujeto histórico, la monarquía, para nada homogéneo, pero que, definitivamente, seguía jugando un papel más o menos real (y continúa aún) dentro de la formación moderna de Europa en el siglo xx. Una vez más, esta naturalización de la monarquía como un único sujeto (trans)histórico que se conjuga a la perfección con los sistemas constitucionales actuales forma parte del mismo (auto)engaño en la lectura de la historia. 
1804) y el Código civil holandés que entró en vigor a principios de 1992. Se puede (y quizá se debe) preguntar, ante esta excepcional retahíla de ejemplos, a) si aún tiene sentido reflexionar sobre el concepto histórico de código, dado que cosas tan distintas son llamadas de la misma manera; b) pero también si, por el contrario, tiene sentido llamar cosas tan distintas de la misma manera; si esta actividad tiene todavía algo en común con lo que acostumbramos a llamar historiografía o no es, acaso, un nuevo juego de sociedad.

En principio, creo que carece de sentido seguir cuestionando el concepto de código, pero definitivamente, utilizarlo puede llevar a naturalizar el derecho transformándolo en algo que existió siempre y que solo se debe retomar, traducir o interpretar según su espíritu para adaptarlo. La clave, entonces, no es pensar la Historia del Derecho (en estos mismos términos) sino en el Derecho en la Historia en tanto expresión cultural con sus condicionantes específicos. En ese sentido, las Siete Partidas son el escenario perfecto para entender el modo de fazer la ley en Castilla en el siglo XIII. Pero, más aún, su larga pervivencia (que llega hasta nuestros días) es muestra viva de los diversos procesos culturales a los que se sometió un texto que fue en sí mismo un fenómeno político medieval. De otro modo deberíamos pensar que las $S P$ de hoy son las mismas que las de 1256-1284, lo cual parece poco probable. Esta condición excepcional de la compilación de $S P$ está determinada por una multimodalidad jurídica, donde el sujeto hablante (sea este Alfonso en tanto mens auctoris o su taller ${ }^{7}$ ) implementa una serie de estrategias discursivas que solo son visibles a través del análisis y que responden no a conceptos tales como importancia, jerarquía o preponderancia, sino a eventualidades que se corresponden con dos cosas. Por un lado, la necesidad comunicacional, que se podría calificar de política ${ }^{8}$. Por otro lado, la condición cultural de los discursos a la mano y vigentes en la época. A este último elemento se suman, dentro del propio discurso jurídico, las fuentes del derecho y sus condicionantes narrativos específicos que interactúan con el discurso unidireccional de la compilación.

\section{Compilar Derecho era hacer derecho}

La práctica a la que refiero es compilar. En tiempos de los griegos ya existía la práctica de «faire des livres avec des libres» (Momigliano 1983: 29). Sin embargo, es la recuperación y producción de textos en la Edad Media lo

\footnotetext{
${ }^{7}$ En general refiero a Alfonso como autor del modo en que lo hace María Rosa Lida (citado en Rico 1984: 11). Igual lo expresa Craddock (1981: 388) y García Solalinde (1915: 283-88).

${ }^{8}$ De algún modo, este punto se corresponde con los análisis de Rodríguez Velasco (2010) y de Craddock (1983) en torno a los cambios y, a la vez, pervivencia relativos a Partidas.
} 
que interesa aquí, ya que esa práctica estuvo determinada por una técnica: la compilación. Guenée (1985: 121) establece el año de 1129 como inicio de la recuperación, aunque cabe aclarar que en su estudio busca el uso del vocablo compilare, compilator, etc. y no la práctica en sí misma. Claro que la práctica concreta de compilar nunca estuvo ausente, pero la conciencia sobre esta relación entre compilar y producir un discurso nuevo se revela a través de la enunciación de la acción y su definición concreta. Ahora bien, se trata entonces de la capacidad y condición, o intención, de innovación que aparece en la Edad Media asociada a la técnica compilatoria.

Si bien existen prólogos anteriores a 1129 que transmiten el vocablo compilare (un ejemplo es el de Anselmo de Lieja, $\dagger$ 1056), no falsean la fecha anterior y su cantidad los hace raros. Sin embargo, es a partir de la Historia Anglorum de Enrique de Huntington que la idea de compilación y compilador quedó instalada en el universo simbólico medieval como sinónimo de producir textos (Guenée 1985: 121). Asimismo, sería en 1214 el momento en que aparece por primera vez la idea de compilator de la mano del anónimo autor de la Historia Regum Francorum (Guenée 1985: 122). En ese momento estaba cristalizada ya la idea de compilar como función positiva y asociada a novedad en la Edad Media ${ }^{9}$. Así, como la concepción sobre la compilación no fue la misma a lo largo de toda la Edad Media, tampoco lo fue la interpretación que los críticos realizaron sobre la misma. Burnyeat (2011) explica que los avances recientes sobre teoría literaria medieval permiten desarrollar nuevas perspectivas que, a su vez, trastocan la manera en la que nosotros mismos leemos la producción textual de aquellos tiempos. A modo de resumen, es posible decir que las perspectivas modernas sobre la compilación medieval comprenden dos posturas. La primera se centra en el rol del proceso compilatorio, en el desarrollo y en la producción de los textos. Estos estudios hacen foco en las collectiones y florilegia que se asocian a auctoritates y a la circulación "generalizada". La segunda, se centra en los efectos producidos por las técnicas de la compilación. Se refiere a cambios físicos o semánticos en la presentación de los textos, diseño, arreglo, estabilización, comentarios marginales, etc. Esta distinción no implica, necesariamente, oposición, sino una interrelación que bien pudo comenzar en el período medieval. Tal y como

\footnotetext{
${ }^{9}$ Vale aclarar que la latinidad clásica no compartía esta idea sobre la compilación. Cicerón y Horacio, por ejemplo, definían compilare como robar, al igual que Papias que decía que compilar es furari, depopulari, expoliare, depilare, etc. En la Alta Edad Media la concepción no cambió demasiado. Isidoro de Sevilla, irónicamente siguiendo a Jerónimo, explicita en sus etimologías que «Conpilator, qui aliena dicta suis praemiscet, sicut solent pigmentarii in pila diversa mixta contundere. Hoc scelere quondam accusabatur Mantuanus ille vates, cum quosdam versus Homeri transferens suis oermiscuiset et conpilator veterum ab aemulis diceretur» (Etym. X, 44).
} 
explica Minnis, en este período comienza un creciente interés de parte de los compilatores por hacerse cada vez más presentes y visibles a través de la reorganización del texto por medio de tabulae, capitulationes, correctoria y concordantiae, etc. (1979: 385). Todo esto no hace sino explicar los cambios en la práctica cultural de hacer libros, fenómeno que no escapa al derecho ${ }^{10}$.

El siglo XIII, la centuria de Alfonso en Castilla, fue testigo de un momento central del desarrollo cultural: se abrió camino de manera positiva la compilación hasta lograr ser una técnica indiferenciada de la creación textual «pura». Martin propuso que existían determinaciones semánticas que gobernaban las operaciones de la compilación, lo que transformaba a esta última en la expresión de un mensaje (1997: 108). Así, la compilación estaba guiada por la búsqueda de la eficacia comunicativa. Martin planteó cinco procedimientos para esta técnica: «Reproduire», «Réunir», «Assembler. Bâtir», «Assembler. Agencer» y «Réviser». El primero refiere a uno muy caro para $S P$ : recostarse en las figuras clásicas de antaño para dar cierto aspecto de insoslayable verdad en los dichos, además de denotar su lectura. Aunque esto puede carecer de valor a primera vista, hay una generación potente de sentido. Por un lado, se refería a algo cuyo sentido ya no tenía vigencia, pero sumado a esa presencia se adosaba una significación nueva, en buena medida dependiente no solo de la traducción, la elección del pasaje o la puesta en diálogo dentro del texto mayor, sino también dentro del espacio de enunciación nuevo que podía darle un marco narrativo diferente. Un ejemplo de esto puede estar dado por el trabajo realizado sobre el De anima de Aristóteles, cuando se lo introdujo en la Segunda Partida con el fin de construir dentro del libro de derecho una representación social donde el rey era alma y corazón del pueblo ${ }^{11}$. Es decir, la relocación produce un sentido diverso por el propio movimiento. Asimismo, las citas de autoridad generan aceptación y a la vez confusión. El texto nuevo se mezcla con el antiguo y parecen ser lo mismo. Así, reproducir provee un marco de legitimación y también eficacia a través de la indistinción tendencial entre escrito «antiguo»y «actual».

Reunir, genera cambios a partir del proceso de elección. La compilación no es una «reedición» y juntar pasajes, libros, extractos y diversos autores,

\footnotetext{
${ }^{10}$ Claro que en su afán de repetir y compilar, la calificación negativa de la práctica compilatoria puede encontrarse hasta el siglo XIII al menos. Por ejemplo, san Buenaventura $(\dagger 1274)$ o Vicente de Beauvais $(\dagger$ 1264) consideraban esencial la distinción entre autor y compilador. Para completar este panorama, que no resulta central a mis objetivos, cfr. Minnis (1984) en términos generales, Wieruszowski (1971: 589-627) para conocer la pervivencia de las opiniones de los clásicos en el siglo xIII, y Quillen (1998) para ver este desarrollo y su impacto en el humanismo, fundamentalmente, italiano.

${ }^{11}$ Vale decir que esa obra posee el tamiz de Avicena y, principalmente, del comentario de Averroes al llegar a manos alfonsíes.
} 
implica elegir qué de cada quién será útil a los efectos de comunicar el mensaje que se intenta construir. En una compilación no hay caos de auctoritates, sino una selección con fines a producir algo nuevo. En este punto, la idea de conservación (mantener el saber antiguo de generación en generación) se transforma en transmisión.

El tercer momento, ensamblar en un marco, implica reducir la diversidad de lo reunido y darle un cuadro preconcebido en el que su presencia tenga sentido y a su vez resulte significativa, lo cual produce una organización sintagmática de los propósitos. Al ensamblar los componentes internos entre sí, imbricar los elementos escogidos y enmarcados es esencial para que tengan un sentido unificado. Esta manera de obrar produce transformaciones en el texto fuente que le quitan toda raigambre con su sentido previo. Este procedimiento produce así un reequilibrio semántico a partir de la sumisión de elementos al propósito comunicativo. Finalmente, el nuevo texto debe ser revisado para conocer si se hizo presente el objeto motivador de la acción en el plano transversal del escrito final. Asimismo, en este mecanismo es posible hallar la modificación puntual o general de ese mismo propósito preexistente. Sobre esto, Alfonso X produce un ejemplo gráfico: las reescrituras de la Primera Partida al calor de los avatares políticos castellanos. Todos esos cambios han sido más que importantes en la transmisión del texto ${ }^{12}$. Esta parte del procedimiento compilatorio implica los potenciales desplazamientos de sentido y reacomodamientos a través de los cuales la compilación tome su forma definitiva.

En resumen, la compilación medieval, lejos de ser collages o puras repeticiones con respeto inquebrantable por los «clásicos», constituyeron el motor de la producción escrita y estuvieron motivadas por determinaciones semánticas con arreglo a propósitos específicos que solo pueden ser determinados a través del análisis situacional de cada producto cultural, sea este teológico, filosófico o jurídico. Todo esto se torna más potente si atendemos al hecho de que existió una virtual indistinción de registros discursivos (que permitió, asimismo, el florecimiento de esta práctica). En rigor, dicha indeterminación de géneros nos invita a reconsiderar las condiciones materiales e intelectuales del hacer derecho en la Edad Media. En particular, al menos, en Castilla. Como dice Martin, desde sus fundamentos semiológicos la compilación no se distingue, más que marginalmente, de una obra original. Pero, más allá de la batalla por los conceptos de autoría u originalidad, lo importante es dejar

\footnotetext{
${ }^{12}$ En Panateri (2021) se establece una condición de interpretación en función de tendencias narrativas dentro de las familias manuscritas existentes y conocidas (sapiencial y legalista como distinción). Asimismo, sobre la cuestión es esencial Craddock (1974, 1981, 1983 y 1986), entre otros.
} 
en claro que lo que primó fue la comunicación de algo nuevo y lo que guió que el trabajo fuera de esta manera y no de otra fue que el modo de lograr eficacia en aquellos tiempos correspondía con esos inevitables procedimientos.

\section{Siete Partidas, un texto único}

A la luz de lo expuesto, es posible elucidar que las formas materiales e intelectuales de producción cultural de la Edad Media hacen imposible considerar una corriente codificatoria, así como la idea de un código estrictamente jurídico y sin relación con la teología, la filosofía, la paremiología, etc. El derecho medieval hispano del siglo XIII implica una variedad de registros discursivos y del saber que tienden a someterse al modo legal. Este modo envuelve en un discurso único un sinfín de géneros y saberes que se adaptan a ese discurso legal, al tiempo que lo condicionan. En ese contexto, las Siete Partidas constituyeron un hito sobresaliente al construir un sistema no solo de conocimiento, sino también de performación social sobre la base de diversos postulados. A los efectos de ese sistema, que es normativo, pero también sociológico, la traducción de una ley romana, la utilización de una fictio legis, la inserción de una historia sapiencial o una construcción analógica de carácter filosófico formaron parte de una misma cosa de manera indistinta. Asimismo, esta relación cultural compleja no solo se cumple en un sentido, sino también en el inverso:

La historia de los orígenes de la prosa en lengua vulgar debe iniciarse dirigiendo la mirada hacia las primeras normas jurídicas que desde finales del XII se redactarán en una lengua comprensible para la mayoría, lo que en las poblaciones reconquistadas supondrá el abandono del latín. En el laconismo de muchos de estos tempranos ejemplos no es fácil descubrir preocupaciones artísticas; sin embargo, los textos jurídicos vernáculos suponen el primer discurso de la prosa medieval en castellano y sin ellos no podrían entenderse desarrollos posteriores con mayor especificidad literaria, desde los libros de leyes a los cantares de gesta o las crónicas. (Lacarra 2013: 28)

Si el derecho medieval elevó la ficción hasta constituirla en uno de los fundamentos de la creatividad jurídica, como señala Rodríguez Velasco, la narrativa medieval dramatiza con frecuencia un conflicto jurídico, como se desprende de la lectura de las primeras colecciones de cuentos que circulan por la $\mathrm{Pe}$ nínsula. Se trata de textos estrechamente relacionadas con el mundo oriental, bien por ser traducción de manuscritos árabes, como el Calila e Dimna y el Sendebar, bien por ser una obra recopilada por un converso, como la Disciplina clericalis. (Lacarra 2013: 32) 
Ahora bien, $S P$ posee el lugar que actualmente la crítica le ha otorgado por derecho propio. Un texto que ha atravesado la historia apareciendo, usualmente, en sus momentos críticos (Rodríguez Velasco 2010: 101), sin duda comportó una doble función de objeto y sujeto en esa misma historia. Esta última función, que utilizo en modo figurado, fue posible gracias al hecho de que (en) Partidas (se) construyó una relación clave entre el libro de derecho como concepto transversal, una teoría jurídica, o legal, propia y la incorporación de otros registros ya mencionados que coadyuvaron al establecimiento de un sistema narrativo propio. Este sistema colocó a la monarquía como elemento de lazo social.

Partidas es entonces un discurso jurídico creativo que asimismo interviene, con plena potencia, en su entorno social inmediato (cualquiera sea este el lugar y el momento). Para finalizar, considero necesario realizar algunos «cortes» que permitan una suerte de estudio entre los pliegues del discurso para ver esas instancias compositivas. Tomaré una serie de leyes que considero de particular riqueza: imagen regia o el deber ser del rey que se encuentra en gran parte de la Segunda Partida (títulos 2-5) ${ }^{13}$. Esta imagen regia no refiere a un tratado de moral política o espejo de príncipes, sino a la inserción lógica del rey dentro de su propia teoría legal. Esta imagen y teoría responden en gran medida a estos fenómenos sintetizados supra donde el préstamo de otros registros discursivos tendió, por medio de la compilación, a construir un discurso sui generis. Como explicita Rodríguez Velasco (2020) se produce un doble cambio: por un lado, el discurso anexado es cambiado para que pase a formar parte del lenguaje y de la ratio jurídicas. Por otro lado, el propio cambio se produce al interior del discurso jurídico que es influenciado por esos préstamos. En esta sección el rey posee un lugar dentro de su propio sistema, que comprende al reino, al pueblo, al corazón y al alma. En ese sentido, comenzaré señalando las relaciones entre las teorías del alma y una legal que la Segunda Partida expone.

En 2.19.3 se define al reino como el lugar regido por el rey. La relación entre ellos es como la del alma y el cuerpo, entidad indivisible, donde el rey es rey en tanto que ejerce la función «natural» de la justicia con derecho ${ }^{14}$. Ahora bien, la justicia como manifestación evidente del ejercicio del derecho que es lo que ese cuerpo y alma debe obrar pone en el centro la figura del

${ }^{13}$ No analizaré todos estos títulos en profundidad, pues su tamaño sería inabarcable dentro de la propuesta. Solo me centraré en algunos con el fin de graficar con mayor potencia el eje de mi argumentación.

${ }^{14} \mathrm{~A}$ efectos de la línea narrativa que desarrollo, dejo de lado el elemento de la naturaleza asociado, también, a la recepción de la Ética y la Política aristotélicas. Al respecto: Martin (2010) y Panateri (2016 y 2019b), entre otros posibles. 
rey, pues se presenta como el agente en la acción descrita. Sin embargo, hay un tercer actor de importancia: el pueblo ${ }^{15}$. En 2.10.1 se define: «Pueblo es el ayuntamiento de todos los omes comunalmente, de los mayores e de los medianos e de los menores. Ca todos son menester». Este armado políticojurídico posee un carácter polimórfico donde convergen diversos elementos. A efectos de mi planteo, propongo salir de la metáfora corporal para arribar a otra idea, la de los sentidos pertenecientes al ámbito del alma como imagen del sistema monárquico alfonsí. El panorama se completa con la idea del rey como «cabeça» y el «reyno» como extremidades:

ca asi como yaze el alma en el coraçon del ome, e por ella vive el cuerpo e se mantiene, así en el rey yaze la justicia que es vida e mantenimiento del pueblo. E bien otrosi el coraçon es uno e por él reciben todos los otros miembros unidad, para ser un cuerpo, bien así todos los del reyno, maguer sean muchos, porque el rey es, e deve ser, uno por eso deven otrosi ser todos unos con él para servirle e ayudarle en las cosas que él ha de fazer. E naturalmente dixeron los sabios que el rey es cabeça de reyno. Ca así como de la cabeça nascen los sentidos porque se mandan todos los miembros del cuerpo, bien asi por el mandamiento que nasce del rey... debe haber acuerdo e acrescentar el reyno onde él es alma e cabeça e ellos miembros (P. 2.1.5).

Estos dos armados retóricos no son contrapuestos, sino complementarios. Asimismo, funcionan como distinciones que en Partidas tienen un correlato directo con la idea de la unidad. Entonces, existe una definición de reino como espacio y una relación con el rey que es como la de alma y cuerpo. Esta relación se sella con la idea de movimiento, de acción aristotélica, donde el alma es lo no-espiritual que permite entender motivación y objetivo (aunque estos pudieran ser entendidos como enteléquicos). El alma entonces es lo que mueve, es principio, y en esa metáfora adquiere la forma concreta de justicia. El pueblo se define como un todo donde participan quienes habitan el reino sin excepción de condición «socioeconómica». Este pueblo funge de sentidos exteriores de ese cuerpo que es el reino. En la otra definición la relación se mueve al alma y al corazón. El rey se define como corazón y ahora el alma reside allí, la analogía se completa con el rey como alma, en tanto sinécdoque, y a la vez corazón. La justicia, una vez más, aparece en plano central y aquí, dice Partidas, yace en el rey. La función se ejerce hacia el cuerpo, el cual es, en esa definición, el pueblo. Así, se construye un cuerpo final, el rey como cabeza de donde nacen los sentidos que mandan a los otros miembros. El rey

\footnotetext{
${ }^{15} \mathrm{Al}$ respecto también se han escrito ríos de tinta. Valdaliso Casanova (2011) proporciona un buen balance y otorga suficientes referencias.
} 
manda según justicia para la sanidad del reino, cuerpo y de los miembros. El pueblo (que también se puede homologar con el reino en sí) debe obedecer para que todo fluya secundum naturam.

La acción compilatoria que se ejerce establece una relación entre la ciencia del alma y la ciencia jurídica alfonsí que hace que el texto deba ser entendido como una entidad donde la relación entre títulos y leyes es la de un programa político y no una sumatoria de definiciones legislativas. En ese sentido, el punto clave es la unidad, la cual refiere, como metáfora final, al libro de derecho (Panateri 2020: 114).

En el sistema narrativo inaugurado por la ley se produce una apropiación donde el alma, como elemento central del sistema intelectivo, es definida jurídicamente. En rigor, esa alma aristotélica, generadora de sentidos, tiene un lugar dentro del cuerpo que es ahora uno jurídico (y político). En definitiva, esa construcción no apunta a convertirse en un tratado de moral política, sino que constituye una teoría filosófica que se incorpora dentro de un libro de derecho para darle mayor eficacia comunicativa a la función jurídica que se ejerce desde ese mismo libro. Es decir, se utiliza para definir no solo al rey, sino al lugar en la sociedad que tiene el rey que hace ese libro y, en consecuencia, define al propio libro. Libro que, de igual modo, es en sí metáfora del cuerpo del rey y donde sus leyes son también sus sentidos por los que gobierna el propio libro, en cuanto a su lógica interna, y en cuanto a su capacidad de operar de modo concreto. El rey es así alma del cuerpo político y alma del cuerpo jurídico. En el primer caso, a través de la justicia que ejerce con sus mandamientos. En el segundo, dada la justicia con derecha razón, en su capacidad de ser lo que anima el propio libro de derecho, en el sentido de que es lex animata de su propia teoría jurídica. El alma es lo que da inicio, movimiento, a los sentidos del cuerpo. Donde el alma se define de un modo material, en el sentido de no-espiritual. En rigor, el alma no es un elemento trascendente sino un elemento-motor del sistema sensitivo (Shiffmann 2011: 3-20, se corresponde con De anima III.10, 433a). Esto es una acción cognitiva que permite creación bajo parámetros culturales de repetición y una interpretación del principio aristotélico de que los sentidos poseen unidad en el corazón (De anima II.6, 418a16-18 y III.2, 425a16), definición que se puede encontrar en Partidas también.

En esta enarratio legal la definición aristotélica bajo parámetros naturales del alma y los sentidos que usa Alfonso X para su sistema generan no solo metáforas y analogías, sino también tropologías políticas que sirven al mismo objetivo. Así, en el lugar donde rey y libro pueden ser igualados, ambos como motores y síntesis del sistema legal y político, se crea un dispositivo 
crucial (el libro) y, así como establece la salud del cuerpo político, también establece la salud del cuerpo jurídico al dictaminar cómo debe transmitirse la propia ley. Cierra el círculo donde la transmisión inalterada del libro de derecho implica trascender parámetros temporales y construir una sola forma acabada de fazer la ley.

En este modelo «naturalista» del alma y los sentidos, el modo en el que el rey se comporta implica o conlleva un reflejo de cómo el reino y el pueblo se deben comportar (que es lo mismo que decir la tendencia por la cual efectivamente se comportarán). Esta relación especular es entre el principio móvil y la operación sensorial de la consecuencia. Esta percepción es una estética, también en su sentido aristotélico, que permite entender la relación entre el «deber ser» de la insignia política y la percepción sensorial expresada por el sistema ${ }^{16}$.

La Segunda Partida prosigue con una serie de títulos que se dedican a componer una teoría de la monarquía en clave jurídica. Desde el título 2 y hasta el 11 todos comienzan con la fórmula «qual deve el rey ser a»o «qual ha de ser el rey a», para luego proseguir con «qual deve el pueblo ser», siempre en relación con el rey o el reino, hasta el título 20 inclusive. De toda esta serie referiré a algunos títulos que conforman una suerte de unidad parcial dentro de la Partida y que se encuentran en diálogo con los elementos desarrollados hasta aquí.

El primero de los títulos está referido a cómo debe ser la relación del rey con Dios. El primer punto de interés es que el conocer al que refiere en realidad apunta al seso, i.e. la capacidad intelectiva del rey y, por relación especular, de los hombres. Esto se relaciona con un tándem de literatura sapiencial y paremiológica referida al buen entendimiento de quien manda y hace las leyes. Hay una serie de conceptos clave que sirven para definir al rey. En ese título destacan dos elementos: primero, la aseveración de que «a Dios se lo conoce a través de sus obras y no por natura». Segundo, la relación equilateral de conceptos que hacen al buen rey, a saber: piedad, merced y mesura. Esta mesura, a su vez, redunda en evitar dos problemas, la sobejanía en todas las cosas y su contraparte inevitable, la mengua, pues «lo que sobra, quita valor». En fin, esta definición del rey se hace constantemente en comparación con Dios, para quien, sostiene, es siempre mayor su piedad que el pecado de los hombres. La ley tercera, cierra con una idea potente: la paridad entre

${ }^{16}$ Aunque el recurso de especularidad, al igual que el «deber ser», como elemento retórico-ideológico del discurso alfonsí fue estudiado por Lacomba (2010) y por Panateri (2015), debo esta reflexión específica a una conferencia de Rodríguez Velasco brindada en el Cambridge's Spanish and Portuguese Virtual Seminar en 2020 . 
piedad y justicia. El título siguiente refiere a los pensamientos del rey dentro del proceso natural que conlleva «concebir», «enunciar»y «ejecutar» lo que está en el corazón. Como es habitual en la narrativa alfonsí, los elementos se relacionan en clave de causa-consecuencia donde uno da paso al otro en diverso orden, en este caso el nivel de publicidad e impacto de las cosas: del pensar al obrar. Primero, «asma los fechos que fazera». Luego, las palabras con que muestra los «fechos»y, finalmente, «aduze a acabamiento lo que piensa». En este punto, donde se define el pensamiento, es donde se define «cómo es el rey en sí mesmo». Este es el lugar, también, donde se plantea la condición natural del pensamiento, aquello que implica lo pasado, lo presente y lo futuro. En la ley 2 aparece nuevamente el corazón, pues el pensamiento nace de allí al igual que la justicia del rey, quien es también corazón (y alma y cabeza). Este pensamiento, sostiene, es importante que no sea con saña. En rigor, $S P$ define muchos inconvenientes en el proceso de pensamiento que no deberían afectar al rey para su buen obrar. De esta enumeración destaco un elemento recurrente: que no haya sobejanía de honra, codicia o vicio. La demasía de esto conlleva la mengua del reino, de su salud y de la propia del rey, en la medida en que se transforma en uso, el uso en costumbre y la costumbre «como en» natura. La referencia jurídica a este aspecto moral es notoria, al punto que provee una fórmula ficcional para cerrar algo también común de la narrativa alfonsí: la «escalera creativa», donde una cosa engendra la otra y el producto es mayor y más importante que aquello que le dio paso. El título cuarto define la palabra, la cual es condición humana solamente: «palabra es donayre que han los homnes solamente». Ahora bien, la palabra debe ser bien dicha, pues refleja lo que está en el corazón. Allí, cataloga buenas y malas palabras, donde todas refieren al mismo concepto: no ser sobejanas. El equilibrio está en que sean convenientes: ni menguadas ni sobejanas, para no convertirse en desconvenientes. Asimismo, aunque las menguadas implican mostrarse por corto de entendimiento, dice Alfonso, peor es hablar de más, pues hacerlo produce mengua de entendimiento (en los otros), es decir de eficacia comunicativa. Sobejanía y mesura aparecen entonces como elementos clave de este armado.

El título 5, finalmente, refiere a las «obras». Naturalmente, reaparecen los mismos tópicos, la mesura y no hacer cosas que sean sobejanas. En esta forma de definir al rey por sus obras, pues al igual que Dios a través de ella se lo conoce, debe cumplir con siete virtudes. tres son reservadas a Dios y cuatro al vivir bien en este mundo. Me centraré en estas últimas y referiré en particular a la cordura que se entiende como prudencia, mesura, y se basa en la conocida política de Aristóteles. Partidas establece una relación directa 
entre esta prudencia y el ver las cosas tal cual son para obrar con justicia según derecho. El resto redundará en los mismos elementos, mesura y evitar la sobejanía. La última virtud, la justicia, es definida como la madre de todo bien, ya que permite que todo viva bien. En este desarrollo, la justicia mantiene al reino, tópico paremiológico utilizado ad nauseam. Para el sistema alfonsí es lo mismo que decir que el rey mantiene el reino, pues la justicia tiene forma de natura en él, en la medida que sus obras, palabras y pensamientos tienen un orden específico que conduce inevitablemente a ello. La ley novena realiza el planteo sobre la ira. Allí se puede encontrar una definición que es una traducción literal del De anima: «la ira es sangre hirviendo alrededor del corazón» (I.1, 30 403b). Esta sangre hirviente no permite al corazón pensar, hablar ni, obviamente, obrar bien, pues genera malquerencia y odium y así sus decisiones son «contra razón», «sin derecho»y «sobejanas». La ira conlleva, entre otras cosas, deseos de venganza, que no permiten obrar según derecho. En este sentido, la imagen regia queda atada, en su condición humana personal, dentro del sistema antes descrito. El rey como corazón que mantiene y moviliza al resto del cuerpo, lo hace a través de la justicia y a través de ella adquiere carácter de alma en esa gran metáfora agregada. Por lo tanto, si el corazón se encuentra anegado de sangre, está incapacitado para ejercer decisiones según derecho, es decir, establecer justicia y, por tanto, incapacitado para ser alma y, asimismo, administrar las almas (la infinidad de ellas ahora) que son el pueblo, los sentidos del cuerpo político. El perdón aparece como atributo necesario del rey y con un carácter natural, pero que debe ser usado según derecho, asimismo basado en la piedad, la merced y la misericordia. En ese razonamiento, el perdón es el resultado de juzgar según derecho, pero con la justa medida, la mesura y sin sobejanía, para arribar al estado de justicia que es el estado propio de la relación equilibrada entre el rey, el pueblo y el reino cuando el primero es corazón y alma y el segundo y el tercero, sentidos y cuerpo de su propia teoría.

Estas operaciones aquí estudiadas no pueden ser reducidas a una corriente codificatoria o una "moda". Ni siquiera a una acción política general «paneuropea», pues no se encuentra en $S P$ nada semejante al resto de aquellas producciones legales que fueron enunciadas al principio. A través de la compilación de registros diversos se integró un sistema total, una ingeniería social con base en el derecho, pero nutrida de filosofía, teología y literatura sapiencial. Todo esto, borrado en sus fronteras y reorganizado bajo la lógica jurídica para ser entendidos en sus propios parámetros lingüísticos. La clave de $S P$ es el lenguaje y no solo su orden; todo se acomoda dentro de un discurso que coloca constantemente al rey en el centro del mismo y lo hace a través de 
una apropiación de conceptos filosóficos y teológicos. Lo hace de un modo donde solo la lectura de conjunto permite entender la solidez del texto legal, pues implica entender la relación tropológica entre el cuerpo legislativo, el cuerpo regio y el cuerpo político donde la ciencia del alma se funde con la teoría jurídica en la medida que, como explicita Bocados (49), por la justicia se aseguran las almas.

\section{Referencias bibliográficas}

Alfonso X, Las Siete Partidas del sabio rey don Alfonso el nono. Nuevamente glosadas por el licenciado Gregorio Lopez del Consejo Real de Indias de su Majestad, Salamanca, 1555 [ed. de Gregorio López en reproducción anastática del Boletín Oficial de Estado, 1974].

AsCHERI, Mario (2003), «Dal diritto comune a la codificazione: quale discontinuità», en Antonio Padoa Schioppa, Gigliola de Renzo Villata y Gian Paolo Messeto, Amicitiae Pignus. Milano: Giuffr, pp. 23-34.

Bermejo Castrillo, Manuel (2013), «Primeras luces de la codificación. El Código como concepto y temprana memoria de su advenimiento», Anuario de Historia del Derecho Español, 83, pp. 9-63.

Black, Anthony (1992), Political Thought in Europe, 1250-1450. Cambridge: Cambridge University Press.

BurnyeAT, Abigail (2011), «Córugud and Compilatio in Some Manuscripts of Táin Bó Cúailnge», en línea: <https://www.ed.ac.uk/literatures-languages-cultures/celtic-scottish-studies/research/internal-projects/orality-text> [consulta: 01/04/2021].

CARONI, Pio (2013), Lecciones de historia de la codificación. Madrid: Universidad Carlos III de Madrid.

Clavero, Bartolomé (1991), Razón de Estado, razón de individuo, razón de historia. Madrid: Centro de Estudios Constitucionales.

CRADDOCK, Jerry (1974), «La nota cronológica inserta en el prólogo de las Siete Partidas. Edición y comentario», Al Andalus, 39, pp. 363-390.

CRADDOCK, Jerry (1981), «La cronología de las obras legislativas de Alfonso X el Sabio», Anuario de Historia del Derecho Español, 51, pp. 365-418.

CradDOCK, Jerry (1983), «Must the King Obey his Laws?», en J. Geary (ed.), Florilegium Hispanicum: Medieval and Golden Age Studies Presented to Dorothy Clotelle Clarke. Madison: Hispanic Seminary of Medieval Studies, pp. 71-79.

CradDock, Jerry (1986), The Legislative Works of Alfonso X «el Sabio». A critical bibliography. London: Grant \& Cutler. 
Crombach, Mechtild (ed.) (1971), Bocados de oro. Bonn: Romanisches Seminar de Universität.

Delgado Echeverría, Jesús (2009), «El "Vidal Mayor”, don Vidal de Canellas y los Fueros de Aragón», Revista de Derecho Civil Aragonés, 15, pp. 11-21.

García Solalinde, Antonio (1915), «Intervención de Alfonso X en la redacción de sus obras», Revista de Filología Española, 2, pp. 283-288.

GuENÉE, Bernard (1985), «L'historien et la compilation un XIII ${ }^{\mathrm{e}}$ siècle», Journal des savants, 1, pp. 119-135.

LaCARra, María Jesús (2013), «De las "fazañas" a las "ficciones legales": textos jurídicos y cuentos orientales», El Cronista del Estado Social y Democrático Social, 40, pp. 28-37.

LACOMBA, Marta (2010), «Réécriture et traduction dans le discours d'Alphonse $\mathrm{X} »$, Cahiers d'études hispaniques médiévales, 33, pp. 27-42.

LinDSAY, Wallace (ed.) (1911), Isidoro de Sevilla, Etymologiarum sive originum. Oxford: Oxford University Press.

Martin, Georges (1997), «Compilation (Cinq procédures fondamentales)», Annexes des Cahiers de linguistique hispanique médiévale, 11, pp. 107-121.

Martin, Georges (2010), «Le concept de "Naturalité" dans les Sept Parties d'Alphonse X le Sage», José Antonio Jara Fuente, Georges Martin e Isabel Alfonso Antón (eds.), Construir la identidad en la Edad Media. Poder y memoria en la Castilla de los siglos VII a XV. Cuenca: Ediciones de la Universidad de Castilla-La Mancha, pp. 145-163.

MinNIs, Alastair (1979), "Late medieval discussions of compilatio and the role of the Compilaton», Beiträge zur Geschichte der deutschen Sprache und Literatur, 101, pp. 385-421.

MinnIs, Alastair (1984), Medieval Theories of Authorship, Philadelphia: University of Pennsylvania Press.

Momigliano, Arnaldo (1983), Problèmes d'historiographie ancienne et moderne. Paris: Gallimard.

PANATERI, Daniel (2016) «Naturaleza y monarquía, la identidad en la Edad Media castellana», Nora Franco et al. (eds.), Territorio, Memoria e Identidades. Buenos Aires: Imhicihu/Conicet.

PANATERI, Daniel (2017), El discurso del rey. El discurso jurídico alfonsí y sus implicancias políticas, Madrid: Dykinson.

PANATERI, Daniel (2019a), «Tradiciones manuscritas, estabilización, promulgación, refundición y compilación en la historia del derecho medieval a partir del caso de las Siete Partidas», Temas Medievales, 27:2, pp. 111-140. 
PANATERI, Daniel (2019b), «Elementos árabo-aristotélicos en la obra jurídica alfonsí. Algunas consideraciones», e-Humanista, 42, pp. 241-252.

PANATERI, Daniel (2020), «El libro de derecho como "bien indisponible". El discurso jurídico alfonsí y su función política», La Corónica: A Journal of Medieval Hispanic Languages, Literatures, and Cultures, 48/2, pp. 103-127.

PANATERI, Daniel (2021), «QQual deve ser el rey en sus palabras? Algunas precisiones sobre las Siete Partidas y su tradición manuscrita», en José Manuel Fradejas Rueda, Ricardo Pichel y Enrique Jerez Cabrero (eds.), Las «Siete Partidas» del Rey Sabio. Una aproximación desde la filología digital y material, Madrid/Frankfurt am Main: Iberoamericana/Vervuert.

Quillen, Carol (1998), Rereading the Renaissance: Petrarch, Augustine and the language of humanism. Ann Arbor: Michigan University Press.

Rico, Francisco (1984), Alfonso el Sabio y la 'General estoria': tres lecciones. Barcelona: Ariel.

Rodríguez Velasco, Jesús (2010), «La urgente presencia de Las Siete Partidas», La Corónica: A Journal of Medieval Hispanic Languages, Literatures, and Cultures, 38:2, pp. 97-134.

Rodríguez Velasco, Jesús (2020), Dead Voice. Law, Philosophy, and Fiction in the Iberian Middle Ages. Philadelphia: University of Pennsylvanya Press.

Shiffmann, Mark (ed. y trad.) (2011), De anima. Newburyfort: Collins.

Valdaliso Casanova, Covadonga (2011), «El pueblo visto desde la Corte. La representación del pueblo en la obra legislativa e historiográfica de Alfonso X, el Sabio», Signum, 12:1, pp. 98-110.

Wieruszowski, Helena (1971), Politics and Culture in Medieval Spain and Italy. Roma: Edizioni di storia e letteratura.

Wolf, Armin (1983), «Los Fori Aragonum de 1247 y el Vidal Mayor. Sus relaciones con la historia de la legislación europea», Anuario de Historia del Derecho Español, 53, pp. 177-203.

WoLF, Armin (1989), «El movimiento de legislación y de codificación en Europa en tiempos de Alfonso el Sabio», en AA.VV., Alfonso X el Sabio, vida, obra y época. Madrid: Sociedad Española de Estudios Medievales.

Wolf, Armin (1993-94), «Los Iuria propria en Europa en el s. XIII», Glossae, 5-6, pp. 35-44. 
$\cos$

\section{El Derecho en la Historia: las Siete Partidas \\ Y SU LUGAR COMO EVENTO CULTURAL}

RESUMEN: En este trabajo se establece que las Siete Partidas son un discurso jurídico particular que se compuso a través de la utilización de diversos registros discursivos con vistas a conformar un sistema narrativo único. Se parte de una crítica a las visiones pan-europeas sobre el derecho a partir de considerar que la creación de Partidas respondió a las condiciones materiales y culturales medievales de composición textual. En función de esto último, se expone la idea de compilación como práctica activa en relación a la creación jurídica. Finalmente, se provee un análisis de algunos títulos de la Segunda Partida con el fin de demostrar la idea central ya expuesta.

Palabras-Clave: Monarquía. Derecho. Discurso. Compilación. Filosofía. Teología.

\section{LaW in History: the Siete Partidas and its Place as a Cultural Event}

ABSTRACT: In this paper, it is established that the Siete Partidas is a specific legal discourse that was composed by different discursive genres in order to build a unique narrative system. The analysis starts from a critique of the pan-European views on Law. In that sense, it is settled that the creation of the Partidas is due to the medieval material and cultural conditions of textual composition. Based on this, the idea of compilation is presented as active practice concerning legal creation. Finally, an analysis of some titles of the Segunda Partida is provided in order to demonstrate the central idea already stated.

KeYwords: Monarchy. Law. Discourse. Compilation. Philosophy. Theology. 